\title{
Clinical Relevance of Cross-Reactive Fungal Allergens
}

Crameri R, Zeller S, Glaser AG, et al.: Cross-reactivity among fungal allergens: a clinically relevant phenomenon? Mycoses 2009, 52:99-106.

Rating: $\bullet$ Of importance.

Introduction: Sensitivity to fungi is a common clinical problem.

Aims: This study was a review of the cross-reactivity of fungal allergens.

Methods: The article draws on fungal allergen protein and DNA sequence databases and on experimental data using in vitro $\mathrm{IgE}$ assays and skin testing to determine the cross-reactivity of various fungal allergens.

Results: Many fungal allergens are cross-reactive and represent conserved proteins in a variety of fungal species.

Discussion: Clinically, many patients exhibit positive skin tests or specific IgE antibodies to a variety of fungal species. Cross-reactivity among fungal allergens may account for these observations.

\section{Comments}

Sensitivity to fungal allergens is an important cause of conditions such as allergic asthma and allergic bronchopulmonary aspergillosis. However, the clinical relevance of cross-reactivity among different fungal species is unknown. Although this is a review article, it reflects an increasing body of knowledge that has evolved through advances in molecular biology. The rapidly expanding DNA sequencing of more than 40 fungal genomes and molecular cloning of several fungal allergens demonstrates that many are crossreactive based on sequence homology and verified by immunologic in vitro and in vivo experiments. The authors of this review are among the world's leading investigators in the molecular characterization of allergens, including fungi. The characterization of fungal allergens shows that cross-reactivity between different fungal species is common and clinically relevant. Continued work in this area undoubtedly will lead to new paradigms and improved diagnostic approaches for fungal allergy.

\section{Acknowledgment}

Dr. Robert K. Bush is affiliated with the University of Wisconsin-Madison, where he is an Emeritus Professor of Medicine. Correspondence should be sent to 600 Highland Avenue, Madison, WI 53792, USA. E-mail: rkb@ medicine.wisc.edu.

\section{Disclosure}

No potential conflict of interest relevant to this article was reported. 